\title{
Carotid endarterectomy for in-stent restenosis: a case report and literature review
}

\author{
BAOFENG XU ${ }^{1}$, RUI LIU ${ }^{2}$, LIQUN JIAO ${ }^{3}$ and JINLU YU ${ }^{1}$ \\ ${ }^{1}$ Department of Neurosurgery, The First Hospital of Jilin University, Changchun, Jilin 130021; \\ ${ }^{2}$ Department of Endocrinology, The Third Hospital of Jilin University, Changchun, Jilin 130021; \\ ${ }^{3}$ Department of Neurosurgery, Xuanwu Hospital of Capital Medical University, Beijing 10053, P.R. China
}

Received May 8, 2017; Accepted June 12, 2017

DOI: $10.3892 /$ br.2017.933

\begin{abstract}
It is relatively difficult to treat restenosis when restenosis occurs following carotid artery stenting. Endarterectomy with simultaneous stent removal is an effective approach when restenosis occurs. The treatment method is still not well known. The present paper reports a case of restenosis after stenting that was treated with endarterectomy with a satisfactory treatment outcome. The patient, a 74-year-old male, underwent stenting 10 months before he was re-hospitalized. He seemed to recover well after stenting until one month before he was re-hospitalized, when he presented symptoms of cerebral ischemia. Imaging examination revealed that in-stent stenosis had reoccurred, with the stenosis degree ranging from 70-99\%. He was given endarterectomy treatment, and intra-operative findings showed that the stent grew into a carotid artery plaque and protruded below the adventitia and that the stent lumen was not covered by carotid artery intima. The surgery completely and successfully removed the plaque and the stent, while the adventitia was completely preserved. Follow-up indicated that there was no stenosis in the carotid artery, and the prognosis was good. Relevant literature was also reviewed in this paper, and the authors suggested that carotid artery plaque and stent endarterectomy is beneficial. However, surgical treatment for in-stent stenosis remains a challenging option.
\end{abstract}

\section{Introduction}

Currently, carotid artery stenting (CAS) remains an effective treatment method for carotid artery stenosis (1). However, following stenting, carotid artery restenosis is still prone to relapse (2). When restenosis after CAS becomes more severe, surgical treatments are most likely required. However, when the

Correspondence to: Professor Jinlu Yu, Department of Neurosurgery, The First Hospital of Jilin University, 71 Xinmin Avenue, Changchun, Jilin 130021, P.R. China

E-mail: jinluyu@hotmail.com

Key words: carotid stenting, carotid endarterectomy, in-stent restenosis stent grows into the carotid artery plaque and even penetrates the carotid artery adventitia, it creates tremendous difficulty for treatment (3). For in-stent restenosis cases, balloon dilatation angioplasty or another stenting method can be feasible treatment options; however, due to the restriction of the existing stent, it is difficult to perform balloon dilatation in a narrow carotid artery; moreover, it is also difficult for the currently used self-expanding stents to maintain the blood vessels at a normal diameter (4). For cases of restenosis after stenting, it is also effective to perform carotid endarterectomy (CEA), a procedure that can remove the stent together with arteriosclerotic plaques, which completely solves the issue of carotid artery stenosis (5). However, because the stent reinforces the plaques and introduces damage to the blood vessel, performing CEA with simultaneous stent removal becomes very difficult. Due to the lack of reports on the treatment of carotid artery restenosis after stenting by CAS, the present paper reports one relevant case that was treated in the Neurosurgery Department of the First Hospital of Jilin University.

\section{Case report}

The patient was a 74-year-old male. At 10 months prior to being re-hospitalized, he underwent a neck blood vessel examination due to 'dizziness and fatigue', and severe stenosis was identified in the left internal carotid artery. Left internal carotid stenting was then performed. A 9x40 mm Acculink self-expanding stent was used, and the stenting process went smoothly. After the stent was released, the imaging demonstrated that the residual stenosis was $<30 \%$. The patient recovered well following the surgery, and his preoperative symptoms disappeared, according to his own statement. After the surgery, he was given aspirin as an anti-platelet aggregation treatment and a long-term oral administration of Lipitor to lower blood cholesterol. The patient was asked to stop smoking and to strictly control his blood pressure. After surgery, the patient was followed up via telephone. The patient had been prescribed medication regularly and stopped smoking, and his blood pressure was stably controlled.

In the last month of follow-up, the patient gradually felt 'somnolence and fatigue', and he returned to the hospital for treatment. A physical examination revealed no positive signs of nervous system involvement. A neck ultrasound indicated 
A

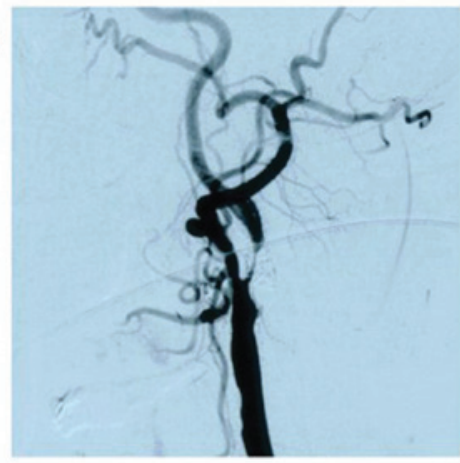

C

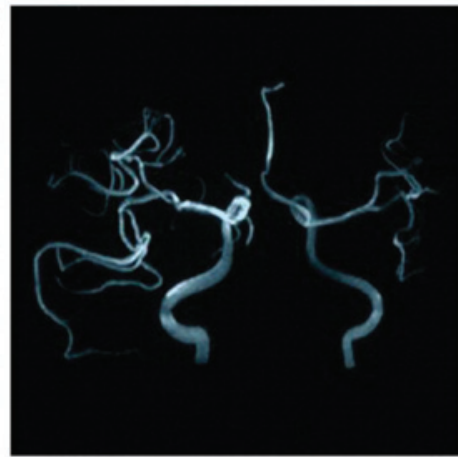

E

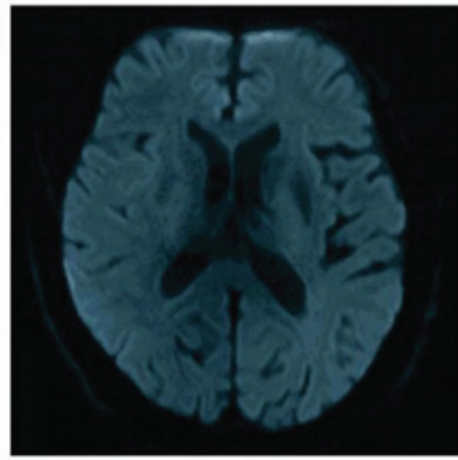

B
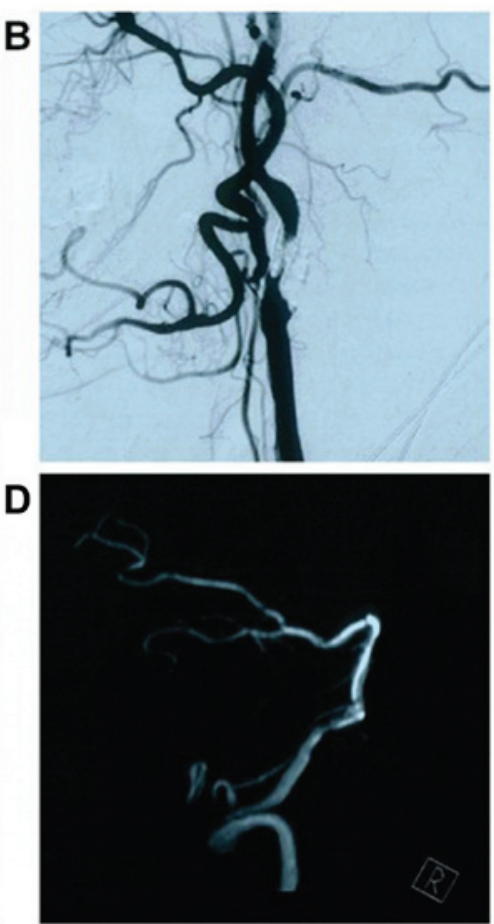

F

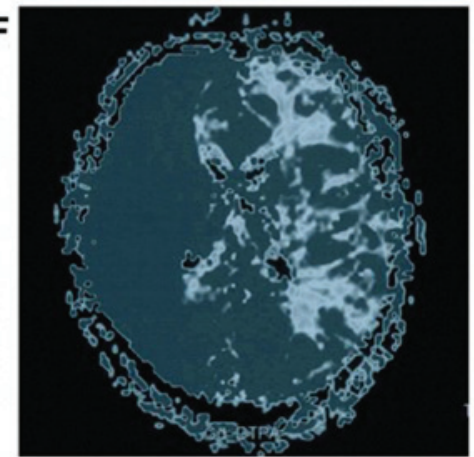

Figure 1. (A and B) Preoperative imaging. Neck digital subtraction angiography presented severe stenosis presenting in the initial segment of the left internal carotid artery, with a stenosis degree of $\sim 90 \%$, and an image of the stent could be seen inside plaques in the left internal carotid artery. (C) Head MRA revealed that the imaging of the intracranial left internal carotid artery system was slightly weaker. (D) Head MRA demonstrated a slight stenosis in the lower basilar artery. (E) Head magnetic resonance imaging did not reveal fresh thrombosis. (F) Head perfusion magnetic resonance indicated that the left cerebral hemisphere had ischemic changes. MRA, magnetic resonance angiography.

that the stent extended from the left common carotid artery to the internal carotid artery, and restenosis appeared in the left internal carotid artery, with a stenosis degree of 70-99\%. The continuity of the lattice-like pattern in the hyperechoic shadow of the stent was poor in two locations. Head magnetic resonance angiography revealed a slightly weaker imaging of the intracranial left internal carotid artery system and a slight stenosis in the lower basilar artery. Magnetic resonance imaging did not present fresh thrombosis, and perfusion magnetic resonance indicated that the left cerebral hemisphere had ischemic changes. Further neck digital subtraction angiography examination revealed severe stenosis in the initial segment of the left internal carotid artery, with a stenosis degree of $\sim 90 \%$. The stent image could be seen inside plaques in the left internal carotid artery (Fig. 1).

At this point, the patient's condition was clearly diagnosed as in-stent stenosis after stenting in the carotid artery, and CEA with simultaneous stent removal was planned. The surgery was performed under general anesthesia, during which a longitudinal incision medial to sternocleidomastoid muscle was used to expose the carotid sheath. After the carotid sheath was opened, the upper edge of the carotid artery stent was visible and palpable through the carotid artery wall. After the common carotid artery, internal carotid artery and external carotid artery were sufficiently exposed, they were temporarily ligated. The common carotid artery and internal carotid artery were incised at the location where the stent remained, and the carotid artery stent together with the carotid arterial intima and media were then peeled off until normal arterial lumen was exposed. The surgery removed the stent and plaques completely, and the adventitia of the common carotid artery and internal carotid artery were shown to be intact. The adventitia was then sutured continuously, and the carotid artery lumen was determined to be unobstructed following suture (Fig. 2). The patient was discharged after good postoperative recovery. After being discharged, the patient received regular dual antiplatelet therapy and lipid-lowering treatment. Half a year following surgery, head computed tomography 
A
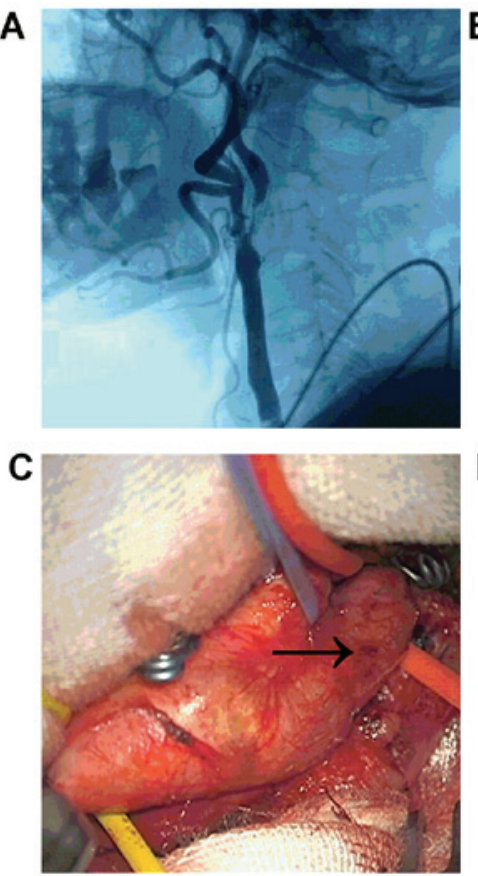

E

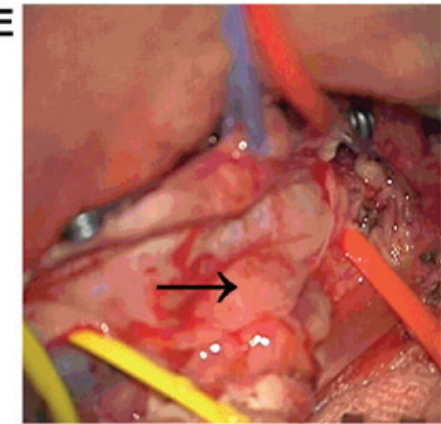

B
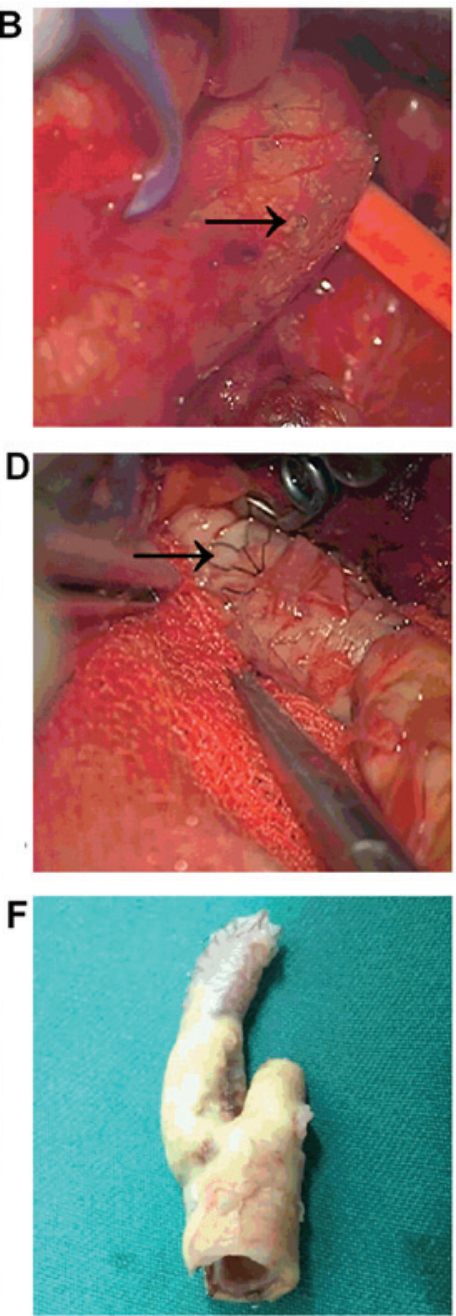

Figure 2. Surgery images. (A) Digital subtraction angiography mask image suggests that the internal carotid artery stenosis was located at the mandibular angle level. (B and C) Following intraoperative exposure of the common carotid artery, the internal carotid artery and the external carotid artery, the stent located in the upper segment of the internal carotid artery can be seen to have penetrated the intima, extending below the adventitia, the black arrow denotes the stent. (D) Following artery dissection, the integrated atherosclerotic plaques and stent were exposed, the black arrow denotes the stent. (E) The plaques and stent were removed completely, indicating that the adventitia of the common carotid artery and internal carotid artery were intact, the black arrow denotes adventitia. (F) An overall view of the removed atherosclerotic plaques and stent.

angiography revealed that the lumen of the internal carotid artery was unobstructed without any stenosis (Fig. 3).

\section{Discussion}

Carotid stenosis in patients is not rare. When its extent reaches severe stenosis $(70-99 \%)$ or a moderate degree of stenosis accompanied by clinical symptoms, patients require surgical treatment; otherwise, the risk for ischemic stroke is very high $(6,7)$. At present, the treatment methods for carotid artery stenosis mainly include CEA and CAS. CEA can remove atherosclerotic plaques, while CAS uses a balloon to dilate the stenosis and then implants a self-expanding stent; both methods are effective methods for treating carotid artery stenosis (8). The results of randomized controlled clinical trials demonstrated that both CEA and CAS could effectively treat carotid artery stenosis and that the differences in complications and long-term prognosis were not significant (9). In the present case, the patient received CAS when the carotid stenosis was initially identified, but he developed in-stent restenosis within 10 months, a shorter interval than previously reported. Rates of in-stent restenosis after CAS vary according to literature sources and the definition of restenosis, ranging from $1 \%$ to $21 \%$ over $12-18$ months (10). There are many causes for in-stent restenosis. For example, in-stent restenosis was more common in an open-cell stent group when compared with a closed-cell stent group after CAS (11). Whether optimal postoperative oral medication treatment was provided, treatment with optimal medical therapy, including anti-platelets and statins, seems to have a beneficial effect on preventing in-stent restenosis in patients undergoing CAS (12). The patient in the present case received the best available postoperative medical therapy, and the risk factors for ischemic stroke were controlled. The Acculink stent used has an open-cell design, supporting the research conclusion that open-cell stents are prone to inducing restenosis.

Restenosis after CAS must be treated. Different treatment methods are available, such as balloon dilatation angioplasty, restenting, CEA with simultaneous stent removal, and carotid bypass reconstruction (13). If an early carotid stenosis 

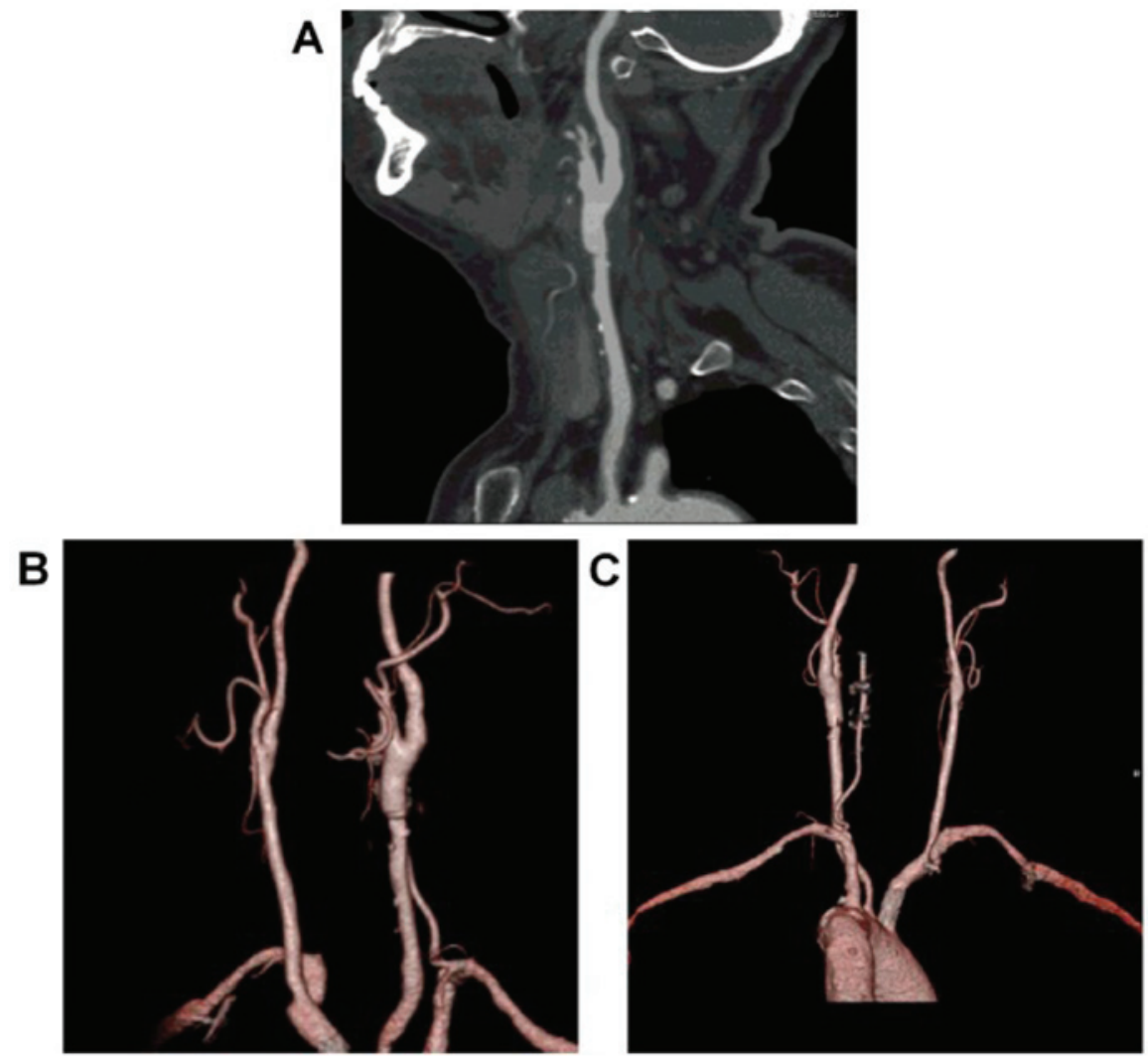

Figure 3. Postoperative computed tomography angiography review. (A) The maximal intensity projection shows good continuity of the common carotid artery and the internal carotid artery without the presence of any stenosis. (B and C) A reconstructed three-dimensional carotid artery shows the blood vessels in the surgical region and the surrounding artery lumen is unobstructed without the presence of any stenosis.

tendency is found after CAS, treatment with cilostazol may be effective (14). If in-stent stenosis is quite severe, it can only be addressed with surgical treatment. For example, in 2014, Gandini et al (15) suggested that drug-eluting balloon dilatation angioplasty for the treatment of refractory recurrent carotid in-stent restenosis has great potential (15). Performing overlap stenting for in-stent restenosis following CAS is also sometimes feasible (16). However, for severe restenosis after stenting, particularly in cases with hard plaques and calcifications, regardless of whether balloon dilatation or stenting is used, both treatment outcomes could be poor. In such a case, CEA may be the only option; in the present case, the patient experienced restenosis after stenting and received CEA with simultaneous stent removal. In 2013, Zheng et al (17) reported one case of endarterectomy following in-stent stenosis occurred. After reviewing previous reports, they suggested that CEA with stent removal in the management of in-stent restenosis was a safe, feasible and effective technique. However, in-stent restenosis after carotid angioplasty and stenting was a challenge for the vascular surgeon (18).

The case reported in this paper describes successfully peeling off the stent, though with great difficulty. After reviewing previous reports in the literature, the authors summarize the treatments as follows. First, the design of the surgical incision should be longer than that of a normal CEA, and the length of the exposure is necessary to dissect out the metallic stent. The upper end of the incision must be extended to the mastoid process along the posterior margin of the parotid gland. Secondly, apart from a larger incision, when dissecting muscles, it should be emphasized that the exposure must be sufficient. When dissecting the sternocleidomastoid muscle, intraoperative incision of the digastric muscle is not necessary, but the sternocleidomastoid muscle must be mobilized towards the mastoid process, and the mobilization of the digastric and sternocleidomastoid muscles must be sufficient so that the distal end of the internal carotid artery can be clearly exposed. Moreover, CEA with stent explantation is more difficult to perform. During the peeling process, because the stent is already immersed in the medium of the carotid artery wall and is only wrapped by adventitia and because plaques have already grown inside the stent, the surface of the stent is not covered by the carotid artery intima. Therefore, when opening and cutting the artery, care is required to remove the stent to avoid vessel wall perforation (19).

For the case reported in this paper, due to careful peeling, the carotid adventitia was preserved completely and was directly sutured. However, when the stent penetrates the carotid artery, CEA may cause defects in the carotid artery, and patches may be needed to repair the defects. For example, the report by Reedy et al mentioned the use of patches (20). Because endarterectomy for the restenosis after stenting is very difficult to perform, the incidence of experiencing complications in the course of the treatment is very high. For example, of the 15 cases reported by Reichmann et al in 2011 (21), one case had minor ischemic stroke, and two cases had neck hematoma; however, the prognoses were all good. It was suggested that CEA with stent explantation for in-stent restenosis after CAS seems effective. 
Based on the present report and this literature review of previous endarterectomy treatments for cases of restenosis after stenting, the authors suggest that CEA of in-stent stenosis following CAS is beneficial. However, surgical treatment for in-stent stenosis remains a challenging option.

\section{References}

1. Calvet D and Mas JL: Recent advances in carotid angioplasty and stenting. Int J Stroke 11: 19-27, 2016.

2. Shankar JJ, Zhang J, dos Santos M, Lesiuk H, Mohan R and Lum C: Factors affecting long-term restenosis after carotid stenting for carotid atherosclerotic disease. Neuroradiology 54 1347-1353, 2012.

3. Gonzalez A, Drummond M, McCord S and Garrett HE Jr: Carotid endarterectomy for treatment of in-stent restenosis. J Vasc Surg 54: 1167-1169, 2011.

4. van Haaften AC, Bots ML, Moll FL and de Borst GJ: Therapeutic options for carotid in-stent restenosis: Review of the literature. J Vasc Interv Radiol 21: 1471-1477, 2010.

5. Akin E, Knobloch K, Pichlmaier M and Haverich A: Instent restenosis after carotid stenting necessitating open carotid surgical repair. Eur J Cardiothorac Surg 26: 442-443, 2004

6. Rosenfield K, Matsumura JS, Chaturvedi S, Riles T, Ansel GM, Metzger DC, Wechsler L, Jaff MR and Gray W; ACT I Investigators: Randomized Trial of Stent versus Surgery for Asymptomatic Carotid Stenosis. N Engl J Med 374: 1011-1020, 2016.

7. Huang JF and Meschia JF: Interventions for Extracranial Carotid Artery Stenosis: An Update. Curr Treat Options Cardiovasc Med 18: 34, 2016

8. Morris S, Patel NV, Dobson J, Featherstone RL, Richards T, Luengo-Fernandez R, Rothwell PM and Brown MM; International Carotid Stenting Study investigators: Cost-utility analysis of stenting versus endarterectomy in the International Carotid Stenting Study. Int J Stroke 11: 446-453, 2016.

9. Howard G, Roubin GS, Jansen O, Hendrikse J, Halliday A, Fraedrich G, Eckstein HH, Calvet D, Bulbulia R, Bonati LH, et al; Carotid Stenting Trialists' Collaboration: Association between age and risk of stroke or death from carotid endarterectomy and carotid stenting: A meta-analysis of pooled patient data from four randomised trials. Lancet 387: 1305-1311, 2016.

10. Sorkin GC, Dumont TM, Eller JL, Mokin M, Hopkins LN, Snyder KV, Siddiqui AH and Levy EI: Instent restenosis after carotid stenting: Treatment using an off-label cardiac scoring balloon. J Vasc Interv Neurol 7: 29-34, 2014.

11. Alparslan B, Nas OF, Eritmen UT, Duran S, Ozkaya G and Hakyemez B: The Effect of Stent Cell Geometry on Carotid Stenting Outcomes. Cardiovasc Intervent Radiol 39: 507-513, 2016.
12. Tendera M, Aboyans V, Bartelink ML, Baumgartner I, Clément D, Collet JP, Cremonesi A, De Carlo M, Erbel R, Fowkes FG, et al; European Stroke Organisation; ESC Committee for Practice Guidelines: ESC Guidelines on the diagnosis and treatment of peripheral artery diseases: Document covering atherosclerotic disease of extracranial carotid and vertebral, mesenteric, renal, upper and lower extremity arteries: the Task Force on the Diagnosis and Treatment of Peripheral Artery Diseases of the European Society of Cardiology (ESC). Eur Heart J 32: 28512906, 2011

13. Bekelis K, Moses Z, Missios S, Desai A and Labropoulos N: Indications for treatment of recurrent carotid stenosis. Br J Surg 100: 440-447, 2013.

14. Yamagami H, Sakai N, Matsumaru Y, Sakai C, Kai Y, Sugiu K, Fujinaka T, Matsumoto Y, Miyachi S, Yoshimura S, et al: Periprocedural cilostazol treatment and restenosis after carotid artery stenting: The Retrospective Study of In-Stent Restenosis after Carotid Artery Stenting (ReSISteR-CAS). J Stroke Cerebrovasc Dis 21: 193-199, 2012.

15. Gandini R, Del Giudice C, Da Ros V, Sallustio F, Altobelli S, D'Onofrio A, Abrignani S, Vasili E, Stanzione P and Simonetti G: Long-term results of drug-eluting balloon angioplasty for treatment of refractory recurrent carotid in-stent restenosis. J Endovasc Ther 21: 671-677, 2014.

16. Nishihori M, Ohshima T, Yamamoto T, Goto S, Nishizawa T, Shimato S, Izumi T and Kato K: Overlap stenting for in-stent restenosis after carotid artery stenting. Nagoya J Med Sci 78: 143-149, 2016.

17. Zheng J, Liu L, Cao Y, Zhang D, Wang R and Zhao J: Carotid endarterectomy with stent removal in management of in-stent restenosis: A safe, feasible, and effective technique. Eur J Vasc Endovasc Surg 47: 8-12, 2014.

18. Setacci C, de Donato G, Setacci F, Pieraccini M, Cappelli A, Trovato RA and Benevento D: In-stent restenosis after carotid angioplasty and stenting: A challenge for the vascular surgeon. Eur J Vasc Endovasc Surg 29: 601-607, 2005.

19. Vale FL, Fisher WS III, Jordan WD Jr, Palmer CA and Vitek J: Carotid endarterectomy performed after progressive carotid stenosis following angioplasty and stent placement. Case report. J Neurosurg 87: 940-943, 1997.

20. Reedy FM, Colonna M, Genovese V, Mancuso M, Napoleone M, Notari P and Di Cintio V: Successful surgical treatment of two patients with restenosis after previous stenting of the carotid artery. Eur J Vasc Endovasc Surg 20: 99-101, 2000.

21. Reichmann BL, van Laanen JH, de Vries JP, Hendriks JM, Verhagen HJ, Moll FL and de Borst GJ: Carotid endarterectomy for treatment of in-stent restenosis after carotid angioplasty and stenting. J Vasc Surg 54: 87-92, 2011. 